\title{
The effect of non-nutritive sweeteners on lipid metabolism in liver cells
}

\author{
Urša Cankar, Saša Kenig \\ University of Primorska, Faculty of Health Sciences, Polje 42, 6310 Izola, Slovenia \\ ursa.cankar1@gmail.com, sasa.kenig@fvz.upr.si
}

\begin{abstract}
Introduction: The use of non-nutritive sweeteners is common in the elderly population, since they often want to avoid sugar intake, as it is also recommended. Additionally, due to the widespread use of sweeteners in a variety of food products, the consumers are sometimes unaware of their consumption. Although they are generally safe to consume, there is also evidence supporting the opposite. In animal models, they have been shown to increase serum triglycerides and fasting insulin levels and interfere with the levels of adipogenic proteins, when consumed regularly. The exact mechanisms are not known. Here, we investigated the effects of the selected sweeteners on the lipid metabolism in human liver cells. Methods: Four sweeteners available on the Slovenian market were selected, namely erythritol, stevia and two commercially available mixed sweeteners Huxol Original (containing sodium cyclamate, sodium saccharin) and Natreen Classic (containing cyclamate, saccharin and thaumatin). Using RTPCR we studied the effect on the expression of genes related to lipid metabolism, and measured the accumulation of OilRed O stained lipid droplets in HepG2 liver cell line exposed to the highest non-cytotoxic concentration of each tested compound for $24 \mathrm{~h}$. All experiments were performed both in the presence and absence of sodium palmitate. Results: Natreen, stevia and erythritol caused an approximately 2 -fold up-regulation of perilipin-2. In addition, Huxol caused more than 8-fold up-regulation of diacyilglycerol-O-acyltranferase, whereas erythritol suppressed its expression. Further, increased expressions of carnitine palmitoyltransferase 1 and 2 were detected for all sweeteners, when palmitate was present in the media. The accumulation of lipid droplets was significantly increased when palmitate was added to the media, but there was no further increase with the addition of the sweeteners. Discussion and conclusions: Treatment of liver cells with the selected
\end{abstract}


non-nutritive sweeteners did not reflect in altered lipid accumulation.

However, the observed changes in gene expression point to their

important impact on lipid metabolism. As genes related to the beta-

oxidation as well as those related to the fat storage were up-regulated,

long-term effects should be further investigated to confirm their safe use

in terms of liver health.

Keywords: non-nutritive sweeteners, fatty liver disease, gene expression

\section{Introduction}

In the elderly population the use of non-nutritional sweeteners is particularly common. The reason lies in the fact that they follow recommendations and avoid sugar intake. On the other hand, it is important for them to keep the food palatable, since sufficient caloric intake is otherwise hard to achieve. Additionally, due to the widespread use of sweeteners in a variety of food products, the consumers are sometimes unaware of the consumption (Sylvetsky and Rother, 2016). In general, sweeteners are safe to consume (Butchko et al., 2002), but there is also evidence supporting the opposite. In contrast to the common expectation, they do not lower body weight and do not even prevent its gain, and glucose levels may also be elevated (Swithers, 2016). Numerous observational studies also suggest an association with metabolic syndrome which includes non-alcoholic fatty liver disease, but there may be a possible association with obesity (Green and Syn, 2019). In animal models, they have been shown to increase serum triglycerides and fasting insulin levels and interfere with the levels of adipogenic proteins, when consumed regularly. Rats which consumed drinks sweetened by aspartame for two months, had increased visceral fat and other characteristics of metabolic syndrome, including hyper-glycemia, increased serum triglycerides and increased low-density and very low-density lipoproteins compared to rats which consumed water (Lebda, 2017). In the same study the levels of leptin were higher, the expression of peroxisomal proliferating receptor decreased and the elevated serum transaminase indicated liver damage. It has been known for a while that sweeteners are not metabolically inert, and further research is needed to elucidate the mechanisms by which sweeteners can promote metabolic effects (Pepino, 2015). In the present study we investigated the effects of the selected sweeteners on the lipid metabolism in human liver cells.

\section{Methods}

Four sweeteners available on the Slovenian market were selected, namely erythritol, stevia and two commercially available mixed sweeteners Huxol Original (containing sodium cyclamate, sodium saccharin) and Natreen Classic (containing cyclamate, saccharin, thaumatin). The analyses were performed in vitro on a HepG2 cell line. HepG2 cells represent a liver cell line derived from human hepatoblastoma, which has been found to express a whole range of liver-spe- 
cific metabolic functions and is therefore an excellent model for studying the central role of the liver in lipid metabolism (Javitt, 1990). The study began with the cultivation of adherent HepG2 liver cells and exposing them to the four different sweeteners to determine cytotoxic concentrations. In the following experiments, the cells were exposed to the highest non-cytotoxic concentration of each tested compound for 24 hours. We examined the effect of sweeteners on the expression of five genes related to lipid metabolism using quantitative polymerase chain reaction (RT-PCR). The genes were: acetyl-CoA carboxylase alpha (ACACA), carnitine palmitoyltransferase $1 \mathrm{~A}(\mathrm{CPT} 1 \mathrm{~A})$, carnitine palmitoyltransferase 2 (CPT2), diacylglycerol O-acyltransferase 2 (DGAT2) and perlipin 2 (PLIN2). We also measured the accumulation of OilRed $\mathrm{O}$ stained lipid droplets in the HepG2 liver cell line. All experiments were performed both in the presence and absence of sodium palmitate.

\section{Results \\ Gene expression analysis}

The cytotoxic effect of selected sweeteners at different concentrations on HepG2 cells after 24 hours of exposure was first studied. All concentrations of erythritol sweetener solution from 0.02 to $2 \mathrm{mg} / \mathrm{ml}$ were found to have a slight proliferative effect on cells. Similar to erythritol, three concentrations of Natreen Classic sweetener solution, $2 \mathrm{mg} / \mathrm{ml}, 1 \mathrm{mg} / \mathrm{ml}$ and $0.2 \mathrm{mg} / \mathrm{ml}$, had a proliferative effect on cells. On the other hand, rebaudioside A (active compound in Stevia Sugarel) at concentrations of $2 \mathrm{mg} / \mathrm{ml}$ and $1 \mathrm{mg} / \mathrm{ml}$ had a cytotoxic effect on cells, while none of the tested concentrations of Huxol Original had either positive or negative effect on cell viability.

Based on the obtained data, further analyses were performed with the highest non-cytotoxic concentration. The following concentrations were used: $2 \mathrm{mg} / \mathrm{mL}$ for Erythritol, Huxol Original and Natreen Classic and $0.2 \mathrm{mg} / \mathrm{ml}$ for Stevia Sugarel. Analysis of fat accumulation-related gene expression was performed for five genes: ACACA, DGAT2, PLIN2, CPT1A and CPT2. Gene expression was tested in cells grown in control medium (source of glucose) and medium with added palmitate (source of glucose and fatty acid). Analysis was performed by RT-PCR, checking the relative expression for each of the selected genes relative to the $18 \mathrm{~S}$ rRNA gene. The results showed increased expression of four of the five genes. ACACA gene expression was below the detection limit in most of the analysed samples.

The results of the CPT1A expression analysis showed that sweeteners, with the exception of one sample (stevia), increased its expression, as shown graphically in Figure 1. The following changes in gene expression occurred between cells in medium with sweeteners and cells in control medium without additives: sweetener Natreen Classic 2.2 \pm 0.7 -fold up-regulation, sweetener Erythritol 3.0 \pm 0.8 -fold up-regulation, sweetener Huxol Original 4.8 \pm 0.7 -fold up-regulation, sweetener Stevia Sugarel $0.7 \pm 0.2$-fold down-regulation. Palmi- 


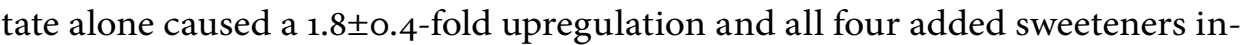
duced further upregulation of the CPT1A. When comparing cells in the medium with palmitate alone and cells with added sweetener and palmitate, Stevia Sugarel showed the most prominent effect. A distinct change in CPT1A expression was observed, while stavia without the presence of palmitate caused a slight downregulation $(\mathrm{F}=0.7 \pm 0.2)$, with the addition of palmitate, CPT1A expression markedly increased $(\mathrm{F}=6.3 \pm 1.1)$.

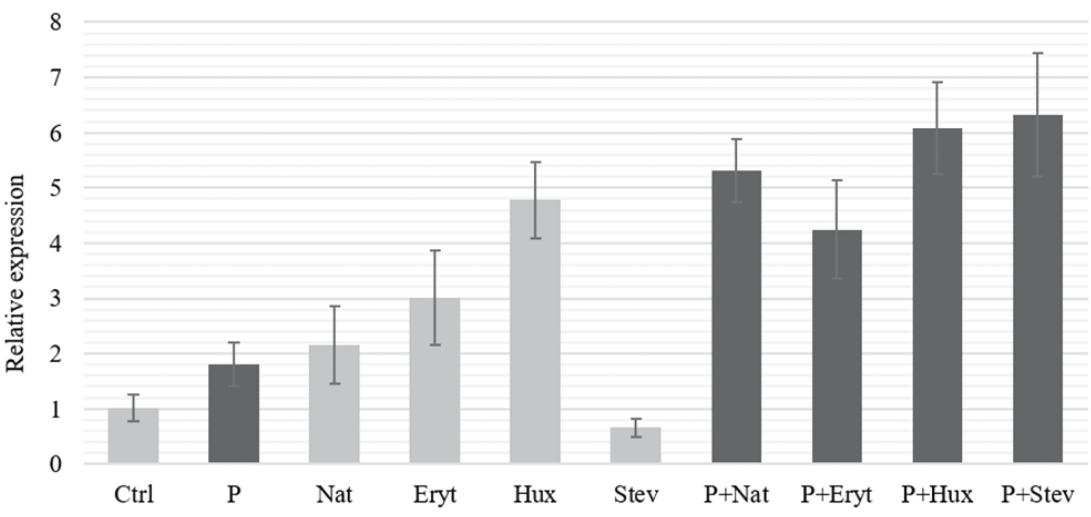

Figure 1: Relative expression of the CPT1A gene in medium, medium with added palmitate, medium with sweeteners and medium with added sweeteners and palmitate. Ctrl-control, P-Na-palmitate, Nat-Natreen Classic, Erit-Erythritritol, Hux-Huxol Original and Stev-Stevia Sugarel

Also, with the exception of one sample, the sweeteners increased the expression of CPT2, as shown in Figure 2. The following changes in gene expression occurred between the samples of sweeteners in the medium and the cells in the control medium: Natreen Classic 1.4 \pm o.1-fold up-regulation, erythritol sweetener 2.3 \pm 0.2 -fold up-regulation, Huxol Original sweetener 2.5 \pm 0.1 -fold up-regulation, Stevia Sugarel sweetener 0.7 \pm 0.3 -fold down-regulation. Comparison of the samples with added sweetener in the presence of palmitate and those with added palmitate alone showed that the expression increased for all four sweeteners. Similar to the expression of CPT1A, Stevia Sugarel alone downregulated the expression but caused an upregulation in the presence of palmitate. The highest effect was observed for Huxol original, where Huxol alone caused approximately 2 -fold upregulation, but in the presence of palmitate, the upregulation by Huxol was 5.7 \pm 0.5 -fold. Palmitate itself had no significant effect, the CPT2 gene upregulation was only 1.2 \pm 0.1-fold. 


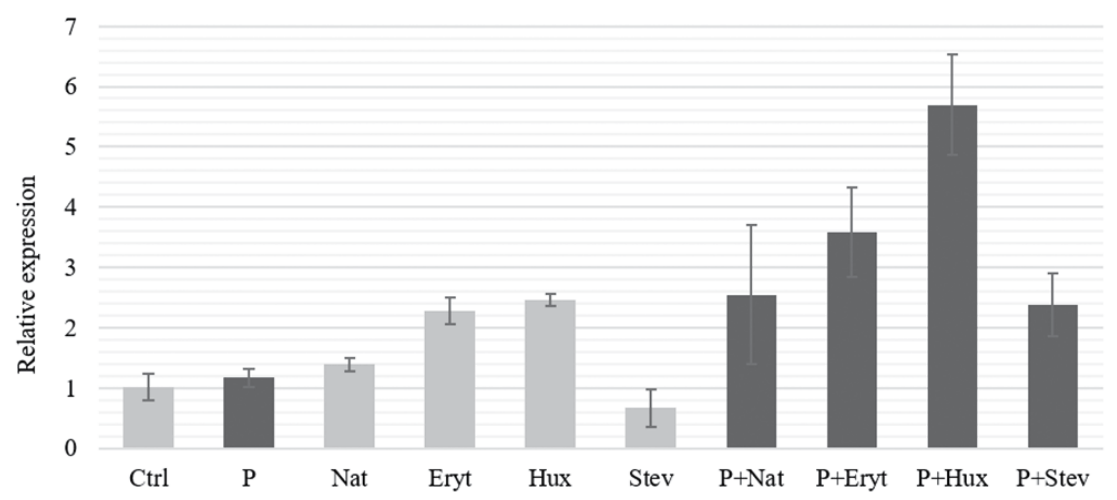

Figure 2: Relative expression of the CPT2 gene in medium, medium with added palmitate, medium with sweeteners and medium with added sweeteners and palmitate. Ctrl-control, P-Na-palmitate, Nat-Natreen Classic, Erit-Erythritritol, Hux-Huxol Original and Stev-Stevia Sugarel

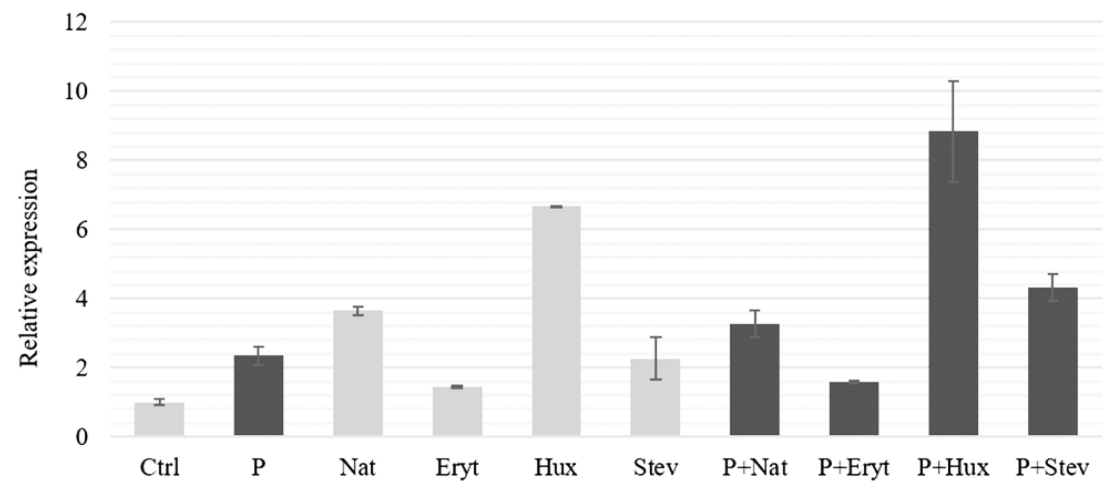

Figure 3: Relative expression of the DGAT2 gene in medium, medium with added palmitate, medium with sweeteners and medium with added sweeteners and palmitate. Ctrl-control, P-Na-palmitate, Nat-Natreen Classic, Erit-Erythritritol, Hux-Huxol Original and Stev-Stevia Sugarel

Analysis of DGAT2 gene expression, presented in Figure 3, showed an increased gene expression with the addition of all four sweeteners. There was the following increase in gene expression between samples of sweeteners in medium and cells in control medium without additives: Natreen Classic 3.6 \pm 0.1 -fold up-regulation, Erythritol sweetener 1.4 \pm 0.0 -fold up-regulation, Huxol sweet-

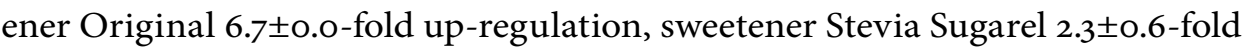
up-regulation. With the addition of the Erythritol sweetener to the palmitate medium (2.4 \pm 0.3 -fold upregulation compared to control), compared to that in

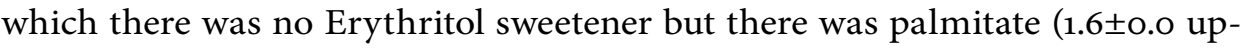
regulation compared to control), we can observe inhibited expression of this gene by this particular sweetener. A similar situation was observed for Stevia 
Sugarel, where gene expression was slightly lower when added to the medium alone $(\mathrm{F}=2.3 \pm 0.6)$ compared to the cells in the palmitate medium $(\mathrm{F}=2.4 \pm 0.3)$, but later an increase of gene expression in medium with sweetener and palmitate $(F=4.3 \pm 0.4)$ occurred. The greatest effect was observed with the sweetener Huxol Original, where there was a $6.7 \pm 0.0$ and $8.8 \pm 1.5$-fold up-regulation in DGAT2 gene expression.

As shown in Figure 4, the sweeteners with the exception of stevia slightly increased the expression of perilipin-2 (PLIN2). The following changes in gene expression occurred between the samples of sweeteners in the medium and the cells in the control medium without additives: in the case of Natreen Classic 1.6 \pm 0.2 -fold up-regulation, Erythritol 2.4 \pm 0.5 -fold up-regulation, Huxol Original 2.2 \pm 0.4 -fold up-regulation, Stevia Sugarel 0.5 \pm 0.1 -fold down-regulation. There was a slight decrease in expression when comparing the cell sample in the medium and the medium with added palmitate, but it was negligible. When cells were grown in the media containing palmitate, the results were similar. The comparison of samples with added sweetener and those with added sweetener and palmitate, there were major changes in the two samples; with the addition of palmitate, the expression of PLIN2 in the sweetener Huxol Original almost halved (from $F=2.2 \pm 0.4$ to $F=1.3 \pm 0.0$ ), which is the only example in our study, where sweetener alone had a stronger effect than the sweetener in combination with palmitate. The opposite occurred with the sweetener Stevia Sugarel, where expression greatly increased (from $\mathrm{F}=0.5 \pm 0.1$ to $\mathrm{F}=$ $2.6 \pm 0.1)$ in the presence of palmitate.

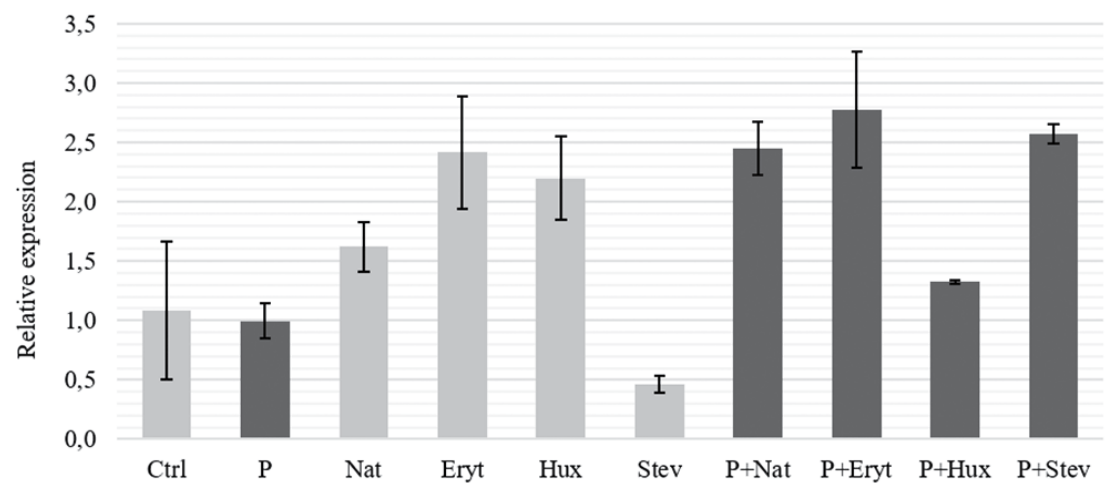

Figure 4: Relative expression of the PLIN2 gene in medium, medium with added palmitate, medium with sweeteners and medium with added sweeteners and palmitate. Ctrl-control, P-Na-palmitate, Nat-Natreen Classic, Erit-Erythritritol, Hux-Huxol Original and Stev-Stevia Sugarel 


\section{Accumulation of lipid droplets}

In the analysis of cell staining with Oil Red $\mathrm{O}$, the amounts and spatial distribution of fat infiltration of visibly stained lipids were observed under a microscope. The spectrophotometrically measured absorbances of the samples are shown in Figure 5. The difference between the cell sample in the control medium and the cells where palmitate was added to the medium is statistically significant, in the later it is $1.9 \pm 0.04$-fold higher $(\mathrm{p}<0.05)$. In line with our expectations, there are no statistically significant differences between the cells in the control medium and the cells where sweeteners are added to the medium, which is also consistent with the previously performed visual analysis of the samples.

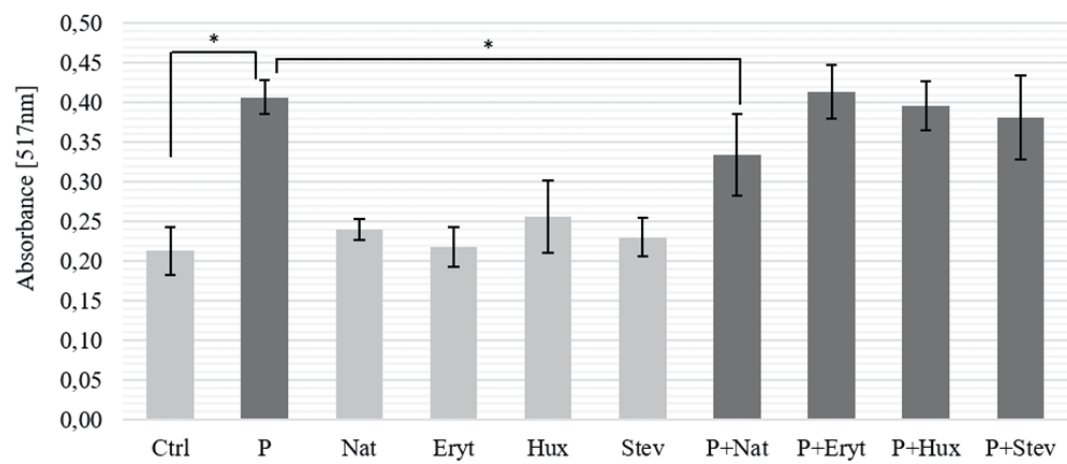

Figure 5: Results of spectrophotometric analysis of fat accumulation in HepG2 cells in the presence of sweetener and sweetener and palmitate. Ctrl-control, P-Na-palmitate, Nat-Natreen Classic, Erit-Erythritritol, Hux-Huxol Original and Stev-Stevia Sugarel, ${ }^{\star} p<0.05$

Significant differences in fat accumulation in samples with added sweeteners Erythritol, Huxol Original and Stevia Sugarel compared to cells where palmitate was added to the cells were not confirmed, but a statistical difference ( $p=0.01$ ) was shown in the treatment of cells with sweetener Natreen Classic. In the case when cells were treated with both Natreen Classic sweetener and palmitate, $0.8 \pm 0.06$-fold lower infiltration of fat into the cells was observed. The results show that the amount of lipid droplets in the cells is significantly increased with the addition of palmitate to the medium compared to the control medium and that the addition of selected sweeteners to the samples in HepG2 cells does not increase fat accumulation in the droplets. In the case of Natreen Classic fat accumulation is even slightly reduced.

\section{Discussion}

In the present study we show that sweeteners stevia, Huxol, Natreen and erythritol have an effect on the expression of genes, related to lipid metabolism. Analysis of gene expression showed that selected sweeteners exert such effect 
in a positive way, as expression of the genes involved in beta-oxidation CPT1A and $\mathrm{CPT}_{2}$ was increased, but also in a negative way, since genes involved in the storage of lipids (DGAT and PLIN2) were upregulated too. These effects, despite some difference, were found for all four studied sweeteners. As expected, the expression of all genes was higher in the presence of palmitate, which served as a source of fatty acids. However, the effect of sweeteners alone was similar also in the environment without added palmitate, where the only fatty acids in the cells are those endogenously produced from glucose.

On the other hand, a functional assay, showing the total accumulation of lipids in hepatic cells, did not confirm any major effect of the sweeteners. In the spectrophotometric analysis of the amount of lipid droplets, we found that the accumulation remained the same or decreased slightly. It should be noted that the analysis of gene expression can only give an estimate that the sweetener acts on a certain mechanism, and that overall accumulation of fat is influenced by other pathways that we have not studied. We conclude that the consumption of selected sweeteners, despite the effect on gene expression, does not affect the increased accumulation of fat in the liver. This is in agreements with the study of Ma et al. (2015), where NAFLD-related markers were not associated with the daily intake of drinks containing non-caloric sweeteners. Further, the investigated sweeteners were found to be non-cytotoxic in tested concentrations, which were calculated from a predicted high daily intake and considering complete absorption, and are thus presumably higher than actual physiologically relevant concentrations. Without a thorough understanding of the absorption and the metabolism of the sweeteners, accurate concentrations of a sweetener in the blood stream reaching liver cells in vivo is difficult to predict.

Although many studies describe the effect of sweeteners on NAFLD and obesity, the mechanisms by which sweeteners affect the human body are not yet entirely clear. According to Schiffman (2012), too little is known about the health effects of sweeteners. They propose the need for further studies in nine different areas, including the need to determine the role of transport molecules in the absorption of sweeteners, the identification of sweetener metabolites, metabolic enzymes and potential toxicity of metabolites, the effect of the sweetener itself on nutrient absorption and weight management, effects of sweeteners on acute and chronical brain activation, neuroplasticity and taste receptor interactions in the brain, checking whether sweeteners have clinically relevant genetic effects, determining long-term effects on the gut microbiota. Of these, only the cytotoxicity and gene-expression analysis were studied here.

Based on the results obtained, we cannot advise against the use of non-caloric sweeteners as an alternative to sugar for NAFLD prevention and other health complications. However, in the analysis of gene expression, we observed significant effects on lipid metabolism that were not previously known. Those are likely not causally related to the development of NAFLD, but confirm that sweeteners are not metabolically inert, as was suggested previously by Pepino (2015) and as such merit further research. 


\section{Conclusions}

Treatment of liver cells with the selected non-nutritive sweeteners did not reflect in altered lipid accumulation. The observed changes in gene expression, however, point to their important impact on lipid metabolism. As genes related to the beta-oxidation as well as those related to the fat storage were up-regulated, long-term effects should be further investigated to confirm their safe use in terms of liver health.

\section{References}

BUTCHKO, H.H., STARGEL, W.W., COMER, C.P., MAYHEW, D.A., BENNINGER, C., BLACKBURN, G.L., DE SONNEVILLE, L.M.J., GEHA, R.S., HERTELENDY, Z., KOESTNER, A. et al., 2002. Aspartame: Review of Safety. Regulatory Toxicology and Pharmacology. April 2002. Vol. 35, no. 2, p. S1-S93. [viewed 13 July 2021]. Available from: http://dx. doi.org/10.1006/rtph.2002.1542.

GREEN, C.H. and SYN, W-K, 2019. Non-nutritive sweeteners and their association with the metabolic syndrome and non-alcoholic fatty liver disease: a review of the literature. European Journal of Nutrition. August 2019. Vol. 58, no. 5, p. 1785-180o. [viewed 13 July 2021]. Available from: http:// dx.doi.org/10.1007/so0394-019-01996-5.

LEBDA, M.A., TOHAMY, H.G. and EL-SAYED, Y.S., 2017. Long-term soft drink and aspartame intake induces hepatic damage via dysregulation of adipocytokines and alteration of the lipid profile and antioxidant status. Nutrition Research. May 2017. Vol. 41, p. 47-55. [viewed 13 July 2021]. Available from: http://dx.doi.org/10.1016/j.nutres.2017.04.002.

MA, J., FOX, C.S., JACQUES, P.F., SPELIOTES, E.K., HOFFMANM, U., SMITH, C.E., McKEOWN, N.M., 2015. Sugar-sweetened beverage, diet soda, and fatty liver disease in the Framingham Heart Study cohorts. Journal of Hepatology, Vol. 63(2), p. 462-469. Available from: https://doi. org/10.1016/j.jhep.2015.03.032

PEPINO, M. Y., 2015. Metabolic effects of non-nutritive sweeteners. Physiology \& Behavior, Vol. 152(Pt B), p. 450-455. Available from: https://doi. org/10.1016/j.physbeh.2015.06.024

SCHIFFMAN, S.S., 2012. Rationale for Further Medical and Health Research on High-Potency Sweeteners. Chemical Senses. October 2012. Vol. 37, no. 8, p. 671-679. [viewed 13 July 2021]. Available from: http://dx.doi. org/10.1093/chemse/bjso53.

SWITHERS, S.E, 2016. Not-so-healthy sugar substitutes? Current Opinion in Behavioral Sciences. June 2016. Vol. 9, p. 106-110. Available from: http:// dx.doi.org/10.1016/j.cobeha.2016.03.003.

SYLVETSKY, A.C. and ROTHER, K.I., 2016. Trends in the consumption of low-calorie sweeteners. Physiology \& Behavior. October 2016. Vol. 164, p. 446-450. [viewed 13 July 2021]. Available from: http://dx.doi. org/10.1016/j.physbeh.2016.03.030 